\title{
Ct Scan And Mri Appearance Of Brain Metastasis In Lung Adenocarcinoma Patients With Epidermal Growth Factor Receptor Mutation Status Evaluation In Dr. Soetomo General Hospital
} Januari 2018 - December 2019

\author{
Timban J.F.J $\mathrm{J}^{\mathrm{a}}$, Erawati $\mathrm{D}^{\mathrm{b}}$, Soeprijanto $\mathrm{B}^{\mathrm{b}}$, Widyoningroem $\mathrm{A}^{\mathrm{b}}$ \\ joanfebry3@gmail.com \\ ${ }^{a}$ Resident, Department of Radiology, Faculty of Medicine Airlangga University, Dr. Soetomo General Academic Hospital Surabaya, \\ Indonesia \\ ${ }^{b}$ Radiologist, Department of Radiology, Faculty of Medicine Airlangga University, Dr. Soetomo General Academic Hospital Surabaya, \\ Indonesia
}

\begin{abstract}
This study aims to find the prevalence and appearance of brain metastasis from CT and MRI in lung adenocarcinoma with negative and positive EGFR mutation status of exon 19 and 21, with evaluation parameters are location, numbers, presence of miliary lesion, largest size, component, contrast enhancement, and adjacent edema. A descriptive, retrospective study was performed on 119 lung adenocarcinoma patient with EGFR mutation status who underwent underwent head CT or MRI during January 2018 until December 2019. We found 19 (55.9\%) patients with positive EGFR mutation status of exon 19; 13 (48.1\%) patients with positive EGFR mutation status of exon 21; and 20 (37.0 \%) patients with negative EGFR mutation status. The CT and MRI of patients with negative and positive EGFR mutation status of exon 19 and 21 have similar appearance, with most common location at corticomedullary, parietal lobe, multiple, with largest size $1.1-2.5 \mathrm{~cm}$ and $2.6 \mathrm{~cm}$, solid-necrotic/cystic component, heterogenous ring contrast enhancement, and perifocal edema
\end{abstract}

Published by IJRP.ORG. Selection and/or peer-review under responsibility of International Journal of Research Publications (IJRP.ORG)

Keywords: lung adenocarcinoma, EGFR mutation, brain metastasis.

\section{Background}

Lung cancer is the leading cause of death in the world with mortality rate around $50 \%$ Yousefi et al., 2017). Adenocarcinoma is the most common type of lung cancer, accounts of more than $40 \%$ of all lung cancer, $60 \%$ of NSCLC and more than $70 \%$ of surgically resected lung cancer (Zeng, 2016). Lung cancer has the potential metastasis to brain even after surgery, radiation, and systemic treatment. The highest incident occurred on the $3^{\text {rd }}$ and $5^{\text {th }}$ year (Mitra et al., 2019). Previous studies stated that most lung adenocarcinoma patients with brain metastasis have positive EGFR mutation status. Lung adenocarcinoma with positive EGFR mutation status of exon 19 showed appearance of brain metastasis lesion with characteristic of small size, large numbers, with minimal adjacent edema. These characteristics are rarely found in brain metastasis of other primary tumors. (Sekine et al., 2012). 


\section{Method}

\subsection{Study design}

This study is a descriptive, retrospective study which performed at Radiology Diagnostic Department in Diagnostic Center Building of Soetomo General Hospital, Surabaya, collecting data of lung adenocarcinoma patient during Januari 2018 - Oktober 2019.

\subsection{Study Population}

All patients with pathology result of adenocarcinoma, with examination result of EGFR mutation status, who underwent brain CT Scan and/or MRI.

\subsection{Data Collecting Procedure}

Data obatained from patients' medical record of Soetomo General Hospital from January $1^{\text {st }}, 2018$ until December $31^{\text {st }}, 2019$ which met the inclusion criterias. The data was brain CT and MRI.

\subsection{Study Protocol}

The researchers identified the medical record data of lung cancer patients, obatain data of lung cancer patients with pathology result of adeocarcinoma and EGFR mutation status examination results, than the raw data of brain CTs and MRIs were evaluated by a neuro-radiologist consultant of Soetomo General Hospital.

\subsection{Study Ethics}

This study was granted an ethical clearance by the Ethical Committee of Universitas Airlangga / RSUD Dr. Soetomo Surabaya.

\section{Result}

We found 119 patients consist of 54 (45.4\%) negative EGFR mutation status (wild type), 34 (28.6 \%) with positive EGFR mutation status of exon 19, 27 (22.7 \%) positive EGFR mutation status of exon 21, 2 $(1.7 \%)$ positive EGFR mutation status of exon 20, and $1(0.8 \%)$ positive EGFR mutation status of exon 19,20. Lung adenocarcinoma patients with positive EGFR mutation status of exon 19 are most common found in female 21 patients $(61.8 \%$ ), while positive EGFR mutation status of exon 21 and negative mutation status most common in male, found in $15(55.6 \%)$ and $40(74.1 \%)$, respectively.

Table 1 : Sample distribution based on EGFR mutation status and gender

\begin{tabular}{lccc}
\hline \multirow{2}{*}{ EGFR Mutation Status } & \multirow{2}{*}{ Frequency } & Male & Gender \\
\cline { 3 - 4 } & $1(0.8 \%)$ & $1(100 \%)$ & $0(0.0 \%)$ \\
Positive of exon 18 & $34(28.6 \%)$ & $13(38.2 \%)$ & $21(61.8 \%)$ \\
Positive of exon 19 & $2(1.7 \%)$ & $0(0.0 \%)$ & $2(100 \%)$ \\
Positive of exon 20 & $27(22.7 \%)$ & $15(55.6 \%)$ & $12(44.4 \%)$ \\
Positive of exon 21 & $1(0.8 \%)$ & $1(100 \%)$ & $0(0.0 \%)$ \\
Positive of exon 19,20 & $54(45.4 \%)$ & $40(74.1 \%)$ & $14(25.9 \%)$ \\
Negative (wild type) & $119(100 \%)$ & $70(58.8 \%)$ & $49(41.2 \%)$ \\
Total & & & \\
\hline
\end{tabular}


Lung adenocarcinoma patients with positive EGFR mutation status of exon 19 and 21 most common in age group 61 years, found in 52.9\% (18 patients) dan 51.9\% (14 patients), respectively, while lung adenocarcinoma patients with negative EGFR mutation status most common in age group 51-60 years, $44.4 \%$ (24 patients).

Table 2 : Sample distribution based on EGFR mutation status and age group

\begin{tabular}{|c|c|c|c|c|c|c|}
\hline \multirow{2}{*}{$\begin{array}{c}\text { EGFR } \\
\text { Mutation } \\
\text { Status }\end{array}$} & \multirow[b]{2}{*}{ Frequency } & \multicolumn{5}{|c|}{ Age group } \\
\hline & & 30 years & $31-40$ years & $41-50$ years & $51-60$ years & 61 years \\
\hline $\begin{array}{l}\text { Positive of } \\
\text { exon } 18\end{array}$ & $1(0.8 \%)$ & $0(0.0 \%)$ & $0(0.0 \%)$ & $0(0.0 \%)$ & $0(0.0 \%)$ & $1(100 \%)$ \\
\hline $\begin{array}{l}\text { Positive of } \\
\text { exon } 19\end{array}$ & $34(28.6 \%)$ & $1(2.9 \%)$ & $4(11.8 \%)$ & $5(14.7 \%)$ & $6(17.6 \%)$ & $18(52.9 \%)$ \\
\hline $\begin{array}{l}\text { Positive of } \\
\text { exon } 20\end{array}$ & $2(1.7 \%)$ & $0(0.0 \%)$ & $0(0.0 \%)$ & $0(0.0 \%)$ & $0(0.0 \%)$ & $2(100 \%)$ \\
\hline $\begin{array}{l}\text { Positive of } \\
\text { exon } 21\end{array}$ & $27(22.7 \%)$ & $0(0.0 \%)$ & $0(0.0 \%)$ & $0(0.0 \%)$ & $13(48.1 \%)$ & $14(51.9 \%)$ \\
\hline $\begin{array}{l}\text { Positive of } \\
\text { exon } 19,20\end{array}$ & $1(0.8 \%)$ & $0(0.0 \%)$ & $0(0.0 \%)$ & $0(0.0 \%)$ & $1(100 \%)$ & $0(0.0 \%)$ \\
\hline $\begin{array}{l}\text { Negative } \\
\text { (wild type) }\end{array}$ & $54(45.4 \%)$ & $3(5.6 \%)$ & $2(3.7 \%)$ & $11(20.4 \%)$ & $24(44.4 \%)$ & $14(25.9 \%)$ \\
\hline Total & $119(100 \%)$ & $4(3.4 \%)$ & $6(5.0 \%)$ & $16(13.4 \%)$ & $44(37.0 \%)$ & $49(41.2 \%)$ \\
\hline
\end{tabular}

Lung adenocarcinoma patient with positive EGFR mutation status of exon 19 are the most with brain metastasis found in 19 patients $(55.9 \%)$. Lung adenocarcinoma patients with positive EGFR mutation status on exon 21 with brain metastasis are 13 patients (48.1\%). Patients with negative EGFR mutation status are 20 patients $(37.0 \%)$.

Table 3 : Sample distribution based on presence of brain metastasis

\begin{tabular}{lcc}
\hline \multirow{2}{*}{ EGFR Mutation Status } & \multicolumn{2}{c}{ Brain metastasis } \\
\cline { 2 - 3 } & Positive & Negative \\
\hline Positif pada exon 18 & $0(0.0 \%)$ & $1(100 \%)$ \\
Positive of exon 18 & $19(55.9 \%)$ & $15(44.1 \%)$ \\
Positive of exon 19 & $0(0.0 \%)$ & $2(100 \%)$ \\
Positive of exon 20 & $13(48.1 \%)$ & $14(51.9 \%)$ \\
Positive of exon 21 & $1(100 \%)$ & $0(0.0)$ \\
Positive of exon 19,20 & $20(37.0 \%)$ & $34(63 \%)$ \\
\hline
\end{tabular}

Lung adeocarcinoma patients who received chemotherapy but have brain metastasis are $62.9 \%(9$ patients) with positive EGFR mutation status of exon 21, 62.2 \% (12 patients) with positive EGFR mutation status of exon 19, and $30.0 \%$ (6 patients) with negative EGFR mutation status. There is also 1 patient with positive EGFR mutation status of exon 19,20 with brain metastasis afeter chemotherapy. 
Table 4. Sample distribution of brain metastasis based on EGFR mutation status and history of chemotherapy

\begin{tabular}{lccc}
\hline \multicolumn{1}{c}{$\begin{array}{c}\text { EGFR Mutation } \\
\text { Status }\end{array}$} & \multirow{2}{*}{ Frequency } & \multicolumn{2}{c}{ History of Chemotherapy } \\
\cline { 3 - 4 } & $19(35.9 \%)$ & $12(63.2 \%)$ & Negative \\
\hline Positive of exon 19 & $13(24.5 \%)$ & $9(69.2 \%)$ & $4(36.8 \%)$ \\
Positive of exon 21 & $20(37.7 \%)$ & $6(30.0 \%)$ & $14(70.0 \%)$ \\
Negative (wild type) & $1(1.9 \%)$ & $1(100 \%)$ & $0(0.0 \%)$ \\
Positive of exon 19,20 & $53(100 \%)$ & $28(52.8 \%)$ & $25(47.2 \%)$ \\
Total & &
\end{tabular}

In this study, $100 \%$ (6 patients) from CT scan and $92.3 \%$ (12 patients) from MRI of lung adenocarcinoma patients with positive EGFR mutation status of exon 19, the lesion located at the corticomedullary layer. Patients with positive EGFR mutation status of exon $21,100 \%$ (6 patients) from CT scan and $71.4 \%$ (5 patients) from MRI located at corticomedullary layer. Patients with negative EGFR mutation status, $90 \%$ (6 patients) from CT scan and $70.0 \%$ (7 patients ) from MRI located at corticomedullary layer, and $10 \%$ (patients)both from CT and MRI located at leptomeningeal layer.

Table 5. Sample distribution of brain metastasis based on EGFR mutation status and location (brain layer)

\begin{tabular}{|c|c|c|c|c|c|c|}
\hline \multirow{3}{*}{ Location } & \multicolumn{6}{|c|}{ EGFR Mutation Status } \\
\hline & \multicolumn{2}{|c|}{ Positive of exon 19} & \multicolumn{2}{|c|}{ Positive of exon 21} & \multicolumn{2}{|c|}{ Negative (wild type) } \\
\hline & CT scan & MRI & CT scan & MRI & CT scan & MRI \\
\hline Corticomedullary & $6(100 \%)$ & $12(92.3 \%)$ & $6(100) \%$ & $5(71.4 \%)$ & $9(90,0 \%)$ & $7(70.0 \%)$ \\
\hline White matter & $0(0.0 \%)$ & $0(0.0 \%)$ & $0(0.0 \%)$ & $1(14.3 \%)$ & $0(0.0 \%)$ & $0(0.0 \%)$ \\
\hline $\begin{array}{l}\text { Corticomedullary } \\
\& \text { white matter }\end{array}$ & $0(0.0 \%)$ & $1(7,7 \%)$ & $0(0.0 \%)$ & $1(14.3 \%)$ & $0(0.0 \%)$ & $2(20.0 \%)$ \\
\hline Leptomeningeal & $0(0.0 \%)$ & $0(0.0 \%)$ & $0(0.0 \%)$ & $0(0.0 \%)$ & $1(10.0 \%)$ & $1(10.0 \%)$ \\
\hline
\end{tabular}

Metastatic lesion location based on brain segment on CT scan, $83.3 \%$ (5 patients) positive EGFR mutation status of exon 19, $66.7 \%$ (4 patients) positive EGFR mutation status of exon 21, and $40.0 \%$ (4 patients) negative EGFR mutation status, located at parietal lobe. From MRI we found $86.4 \%$ (11 patients) positive EGFR mutation status of exon 19, $85.7 \%$ (6 patients) positive EGFR mutation status of exon 21, and $40.0 \%$ (4 patients) negative EGFR mutation status, located at parietal lobe.

Table 6. Sample distribution of brain metastasis based on EGFR mutation status and location (brain segment)

\begin{tabular}{|c|c|c|c|c|c|c|}
\hline \multirow{3}{*}{ Location } & \multicolumn{6}{|c|}{ EGFR Mutation Status } \\
\hline & \multicolumn{2}{|c|}{ Positive of exon 19} & \multicolumn{2}{|c|}{ Positive of exon 21} & \multicolumn{2}{|c|}{ Negative (wild type) } \\
\hline & CT scan & MRI & CT scan & MRI & CT scan & MRI \\
\hline Frontal & $2(33.3 \%)$ & $8(61.5 \%)$ & $2(33.3 \%)$ & $1(14.3 \%)$ & $1(10.0 \%)$ & $7(70.0 \%)$ \\
\hline Parietal & $5(83.3 \%)$ & $11(84.6 \%)$ & $4(66.7 \%)$ & $6(85.7 \%)$ & $4(40.0 \%)$ & $9(90.0 \%)$ \\
\hline Temporal & $3(50.0 \%)$ & $6(46.2 \%)$ & $1(16.7 \%)$ & $1(14.3 \%)$ & $2(20.0 \%)$ & $5(50.0 \%)$ \\
\hline Occipital & $2(33.3 \%)$ & $8(61.5 \%)$ & $3(50.0 \%)$ & $2(28.6 \%)$ & $3(30.0 \%)$ & $7(70.0 \%)$ \\
\hline Basal ganglia & $1(16.7 \%)$ & $2(15.4 \%)$ & $1(16.7 \%)$ & $0(0.0 \%)$ & $1(10.0 \%)$ & $0(0.0 \%)$ \\
\hline
\end{tabular}




\begin{tabular}{lllllll}
\hline Thalamus & $1(16.7 \%)$ & $1(7.7 \%)$ & $0(0.0 \%)$ & $1(14.3 \%)$ & $0(0.0 \%)$ & $0(0.0 \%)$ \\
Midbrain & $0(0.0 \%)$ & $0(0.0 \%)$ & $0(0.0 \%)$ & $1(14.3 \%)$ & $0(0.0 \%)$ & $0(0.0 \%)$ \\
Pons & $0(0.0 \%)$ & $2(15.4 \%)$ & $0(0.0 \%)$ & $0(0.0 \%)$ & $0(0.0 \%)$ & $0(0.0 \%)$ \\
Cerebellum & $2(33.3 \%)$ & $5(38.5 \%)$ & $1(16.7 \%)$ & $1(14.3 \%)$ & $3(30.0 \%)$ & $5(50.0 \%)$ \\
\hline
\end{tabular}

Numbers of metastatic lesion in this study are differentiate between single or 1 lesion and multiple or more than 1 lesions. From CT scan, we found $83.3 \%$ (5 patients) positive EGFR mutation status of exon 19 , $83.3 \%$ (5 patients) positive EGFR mutation status of exon 21 , and $50.0 \%$ (5 patients) negative EGFR mutation, have multiple lesions. From MRI we found, $76.9 \%$ (10 patients) positive EGFR mutation status of exon 19 and $90.0 \%$ (9 patients) negative EGFR mutation status, have multiple lesions, while positive EGFR mutation status of exon $2157.1 \%$ (4 patients) have a single lesion.

Table 7. Sample distribution of brain metastasis based on EGFR mutation status and number(s) of lesion

\begin{tabular}{llccccc}
\hline \multirow{2}{*}{$\begin{array}{c}\text { Number(s) } \\
\text { of lesion }\end{array}$} & \multicolumn{5}{c}{ EGFR Mutation Status } \\
\cline { 2 - 7 } & \multicolumn{2}{c}{ Positive of exon 19 } & \multicolumn{2}{c}{ Positive of exon 21 } & \multicolumn{2}{c}{ Negative (wild type) } \\
\hline \multirow{2}{*}{ Single } & CT scan & MRI & CT scan & MRI & CT scan & MRI \\
Multiple & $1(16.7 \%)$ & $3(23.1 \%)$ & $1(16.7 \%)$ & $4(57.1 \%)$ & $5(50.0 \%)$ & $1(10.0 \%)$ \\
\hline
\end{tabular}

Brain metastatic lesion from CT scan of patients with positive EGFR mutation status of exon 19 size $1.1-2.5 \mathrm{~cm}$ found in $66.7 \%$ (4 patients), patients with positive EGFR mutation status of exon 21 size $2.6 \mathrm{~cm}$ found in $50.0 \%$ (3 patients), and in patients with negative mutation status we found largest size $2.6 \mathrm{~cm}$ found in $50.0 \%$ (5 patients). From MRI of patients with positive EGFR mutation status of exon 19 size $2.6 \mathrm{~cm}$ found in $61.5 \%$ (8 patients), patients with positive EGFR mutation status of exon 21 size $2.6 \mathrm{~cm}$ and 1.1-2.5 $\mathrm{cm}$ found in $42.9 \%$ ( 8 patients) each, and in patients with negative mutation status we found largest size 2.6 $\mathrm{cm}$ found in $70.0 \%$ (7 patients).

Table 8. Sample distribution of brain metastasis based on EGFR mutation status and largest size

\begin{tabular}{|c|c|c|c|c|c|c|}
\hline \multirow{3}{*}{ Largest size } & \multicolumn{6}{|c|}{ EGFR Mutation Status } \\
\hline & \multicolumn{2}{|c|}{ Positive of exon 19} & \multicolumn{2}{|c|}{ Positive of exon 21} & \multicolumn{2}{|c|}{ Negative (wild type) } \\
\hline & CT scan & MRI & CT scan & MRI & CT scan & MRI \\
\hline $1 \mathrm{~cm}$ & $0(0.0 \%)$ & $2(15.4 \%)$ & $1(16.7 \%)$ & $1(14.3 \%)$ & $0(0.0 \%)$ & $0(0.0 \%)$ \\
\hline $1.1-2.5 \mathrm{~cm}$ & $4(66.7 \%)$ & $3(23.1 \%)$ & $2(33.3 \%)$ & $3(42.9 \%)$ & $4(40.0 \%)$ & $2(20.0 \%)$ \\
\hline $2.6 \mathrm{~cm}$ & $2(33.3 \%)$ & $8(61.5 \%)$ & $3(50.0 \%)$ & $3(42.9 \%)$ & $5(50.0 \%)$ & $7(70.0 \%)$ \\
\hline Immeasurable & $0(0.0 \%)$ & $0(0.0 \%)$ & $0(0.0 \%)$ & $0(0.0 \%)$ & $1(10.0 \%)$ & $1(10.0 \%)$ \\
\hline
\end{tabular}

Metastatic lesion component found on CT scan of lung adenocarcinoma patients with positive EGFR mutation status of exon 19 and positive EGFR mutation status of exon 21, all have solid component, while $90.0 \%$ (9 patients) with negative EGFR mutation status have solid component. Cystic component often found from MRI examination, which are $38.5 \%$ (5 patients) positive EGFR mutation status of exon 19, 42.9 \% (3 patients) positive EGFR mutation status of exon 21, and $60.0 \%$ (6 patients) negative EGFR mutation status. Necrotic component often found from CT scan, $50.0 \%$ (3 patients) positive EGFR mutation status of exon 1, $66.7 \%$ (4 patients) positive EGFR mutation status of exon 21 , and $70.0 \%$ (7 patients) negative EGFR 
mutation status. Calcification found on CT scan of $16.7 \%$ (1 patient) with positive EGFR mutation status of exon 21 .

Table 9. Sample distribution of brain metastasis based on EGFR mutation status and lesion component

\begin{tabular}{llccccc}
\hline \multirow{2}{*}{$\begin{array}{c}\text { Lesion } \\
\text { component }\end{array}$} & \multicolumn{5}{c}{ EGFR Mutation Status } \\
\cline { 2 - 6 } & \multicolumn{2}{c}{ Positive of exon 19 } & \multicolumn{2}{c}{ Positive of exon 21 } & \multicolumn{2}{c}{ Negative (wild type) } \\
\hline Solid & \multicolumn{1}{c}{ CT scan } & \multicolumn{1}{c}{ MRI } & CT scan & \multicolumn{1}{c}{ MRI } & CT scan & MRI \\
Necrotic & $6(100 \%)$ & $13(100 \%)$ & $6(100 \%)$ & $7(100 \%)$ & $9(90.0 \%)$ & $9(90.0 \%)$ \\
Cystic & $3(50.0 \%)$ & $2(15.4 \%)$ & $4(66.7 \%)$ & $1(14.3 \%)$ & $7(70.0 \%)$ & $0(0.0 \%)$ \\
Blood & $1(16.7 \%)$ & $5(38.5 \%)$ & $0(0.0 \%)$ & $3(42.9 \%)$ & $2(20.0 \%)$ & $6(60.0 \%)$ \\
Calcification & $2(33.3 \%)$ & $3(23.1 \%)$ & $2(33.3 \%)$ & $0(0.0 \%)$ & $2(20.0 \%)$ & $1(10.0 \%)$ \\
\hline
\end{tabular}

Most of contrast enhancement of lung adenocarcionoma with positive EGFR mutation status of exon 19 from CT scan is ring enhancement in 50.0\% (3 patients) and from MRI is heterogenous in $53.8 \%$ (7 patients). In patients with positive EGFR mutation status of exon 21, both CT andd MRI the most common enahncement pattern is heterogenous contrast enhancement, $50.0 \%$ (3 patients) and 71.4\% (5 patients), respectively. In patients with negative EGFR mutation status from CT scan we found $40.0 \%$ (4 patients) with heterogenous contrast enhacement and $40.0 \%$ (4 patients) with ring contrast enhancement, while MRI examination showed the most common enhancement pattern is ring enhancement found in $40.0 \%$ (4 patients).

Tabel 10. Sample distribution of brain metastasis based on EGFR mutation status and contrast enhancement

\begin{tabular}{|c|c|c|c|c|c|c|}
\hline \multirow{3}{*}{$\begin{array}{c}\text { Contrast } \\
\text { enhancement }\end{array}$} & \multicolumn{6}{|c|}{ EGFR Mutation Status } \\
\hline & \multicolumn{2}{|c|}{ Positive of exon 19} & \multicolumn{2}{|c|}{ Positive of exon 21} & \multicolumn{2}{|c|}{ Negative (wild type) } \\
\hline & CT scan & MRI & CT scan & MRI & CT scan & MRI \\
\hline Heterogenous & $2(33.3 \%)$ & $7(53.8 \%)$ & $3(50.0 \%)$ & $5(71.4 \%)$ & $4(40.0 \%)$ & $3(30.0 \%)$ \\
\hline Homogenous & $1(16.7 \%)$ & $2(15.4 \%)$ & $0(0.0 \%)$ & $0(0.0 \%)$ & $1(10.0 \%)$ & $0(0.0 \%)$ \\
\hline Ring & $3(50.0 \%)$ & $3(23.1 \%)$ & $2(33.3 \%)$ & $2(28.6 \%)$ & $4(40.0 \%)$ & $4(40.0 \%)$ \\
\hline $\begin{array}{l}\text { Ring }+ \\
\text { heterogenous }\end{array}$ & $0(0.0 \%)$ & $0(0.0 \%)$ & $1(16.7 \%)$ & $0(0.0 \%)$ & $0(0.0 \%)$ & $2(20.0 \%)$ \\
\hline $\begin{array}{l}\text { Ring }+ \\
\text { homogenous }\end{array}$ & $0(0.0 \%)$ & $1(7.7 \%)$ & $0(0.0 \%)$ & $0(0.0 \%)$ & $0(0.0 \%)$ & $0(0.0 \%)$ \\
\hline Leptomeningeal & $0(0.0 \%)$ & $0(0.0 \%)$ & $0(0.0 \%)$ & $0(0.0 \%)$ & $1(10.0 \%)$ & $1(10.0 \%)$ \\
\hline
\end{tabular}

Patients with positive EGFR mutation status of exon 19 have the most metastatic lesion with perifocal edema, found in $66.7 \%$ (4 patients) from CT and $38.5 \%$ (5 patients) from MRI. Patients with positive EGFR mutation status of exon 21 , we found $50.0 \%$ (3 patients) with perifocal edema of metastatic lesion from CT scan while $42.9 \%$ (3 patients) with adjacent tentacle edema. In negative EGFR mutation status, from CT we found $60.0 \%$ (6 patients) and from MRI $50.0 \%$ (5 patients) with metastatic lesion and perifocal edema. 
Tabel 11. Sample distribution of brain metastasis based on EGFR mutation status and adjacent edema

\begin{tabular}{llllllc}
\hline \multirow{2}{*}{$\begin{array}{c}\text { Adjacent } \\
\text { edema }\end{array}$} & \multicolumn{7}{c}{ EGFR Mutation Status } \\
\cline { 2 - 7 } & \multicolumn{2}{c}{ Positive of exon 19 } & \multicolumn{2}{c}{ Positive of exon 21 } & \multicolumn{2}{c}{ Negative (wild type) } \\
\hline CT scan & \multicolumn{1}{c}{ MRI } & \multicolumn{1}{c}{ CT scan } & MRI & \multicolumn{1}{c}{ CT scan } & MRI \\
\hline Perifocal & $4(66.7 \%)$ & $5(38.5 \%)$ & $3(50.0 \%)$ & $2(28.6 \%)$ & $6(60.0 \%)$ & $5(50.0 \%)$ \\
Tentacle & $1(16.7 \%)$ & $3(23.1 \%)$ & $0(0.0 \%)$ & $3(42.9 \%)$ & $1(10.0 \%)$ & $1(10.0 \%)$ \\
Negative & $1(16.7 \%)$ & $4(30.8 \%)$ & $2(33.3 \%)$ & $2(28.6 \%)$ & $3(30.0 \%)$ & $3(30.0 \%)$ \\
$\begin{array}{l}\text { Perifocal }+ \\
\text { negative }\end{array}$ & $0(0.0 \%)$ & $0(0.0 \%)$ & $1(16.7 \%)$ & $0(0.0 \%)$ & $0(0.0 \%)$ & $1(10.0 \%)$ \\
$\begin{array}{l}\text { Tentacle }+ \\
\text { negative }\end{array}$ & $0(0.0 \%)$ & $1(7.7 \%)$ & $0(0.0 \%)$ & $0(0.0 \%)$ & $0(0.0 \%)$ & $0(0.0 \%)$ \\
\hline
\end{tabular}

\section{Discussion}

Patients distribution in these study is almost similar to the study by Cruz et al. in 2011 where male distribution of lung cancer is higher than female and the age group is 65-74 years. Study by Sekine et al in 2012 also found that female is significantly higher numbers of lung adenocarcinoma with positive EGFR mutation status of exon 19.

Lung adenocarcinoma patients with brain metastasis, we found $55.9 \%$ (19 patients) with positive EGFR mutation status of exon 19, $48.1 \%$ (13 patients) with positive EGFR mutation status of exon 21, and $37.0 \%$ (20 patients) with negative EGFR mutation status. There is 1 patient with positive EGFR mutation status of exon19,20 also has brain metastasis. Study conducted by Lin et al in 2017 also found that lung adenocarcinoma with EGFR mutation tends to metastasis in brain compared to lung adenocarcinoma with negative EGFR mutation or wild type.

Study by Mitra et al in 2019 found that lung adenocarcinoma with EGFR mutation significantly caused brain metastasis after multimodality therapy with incidence approximately $33 \%$ after 3 years therapy and $43.8 \%$ after 5 years therapy. Thhis study also found that more than half patients with positive mutation status on exon 19 and 21 have brain metastasis even after received chemotherapy but we did not analyze the chemotherapy history further.

Location of brain metastasis lesion, according to literature (Takamori et al, 2018) often found in cortico-medullary area due to constricted and acute angles of vessels. Central location of brain metastasis more likely due to significant damage of BBB. According to literature (Takei et al., 2016), leptomeningeal metastasis is a complication of tumor spread damaging the arachnoid sapce, found in 4-7 \% patients with solid tumor.

Based on brain segment, metastatic lesion from CT and MRI of patients with negative and positive EGFR mutation status of exon 19 and 21, most found in parietal lobe. Study by Pope in 2018 conducted on NSCLC patients also found the most common location of brain metastasis is parietal and occipital lobe.

Numbers of brain metastasis lesion in this study is differentiate between single or one lesion and multiple or more than one lesion, found in patients with negative and positive EGFR mutation status of exon 19 mostly multiple, while in patients with positive EGFR mutation status of exon 21 mostly single. This result is different from the study by Sekine et.al in 2012 which stated that brain metastatis lesion of lung adenocarcinoma in patients with positive EGFR mutation status of exon 19 have a characteristic of multiple lesion compared with patients with negative and positive mutation status of exon 21 . The discrepancy may be due to some patients in this study already received chemotherapy.

Study by Sekine et al in 2012 found that metastasis lesion of patients with positive EGFR mutation status of exon 19 is smaller compared to patients with negative EGFR mutation status. But from this study 
and a study by Takano et al. in 2016 found that size of brain metastasis lesion showed no significant difference between EGFR mutation status of lung adenocarcinoma. The study by Shin $e t$ al in also found no association between lesion size and subtype of EGFR mutation status

According to literature (Oshiro et al, 2008) stated that various signal intensity of brain lesion showed variations of components. This is due to the histological component of the tumor. According to Lin et al in 2019 , necrotic is a result of neovascularisation and rapid growth of tumor followed by lack of blood suply lead to hypoxia of tumor tissue. The hemorrhage in brain metastasis lesion, according to Yoo et al in 2010 caused by numbers of factors including endothelial proliferation with vascular obliteration, tumor compression to vessels, vessels necrosis and tumor invasion to vessel wall. Calcified component found mostly from CT of patients with positive EGFR mutation status of 21, 16.7\% (1 patient). In the literature (Bahrami et al., 2019) calcification are scarcely found in metastasis lesion, only $1.1 \%$ found during surgery and $6.6 \%$ during autopsy. But some case of brain metastasis with calcified component from lung adenocarcinoma.

Contrast enhancement in patients with negative and positive EGFR mutation status of exon 19 and 21, mostly are ring and heterogenous enhancement. According to literature (Lignelli and Khandji, 2011), contrast enhancement is a result from lack of BBB. The enhancement pattern varied accoring to the primary tumor. Other literature (Pope, 2018) stated that ring enhancement pattern found in tumor rapidly outgrew the blood supply lead to necrosis of tumor center.

Study by Sekine et al in 2012 found that brain metastasis lesion with minimal adjacent edema mostly found in lung adenocarcinoma with positive EGFR mutation status of exon 19. In this study we found adjacent edema in patients with negative and positive EGFR mutation status of exon 19 and 21 mostly are perifocal edema. The discrepancy of this study and previous study maybe due to the population of this study is old cases who already received chemotherapy.

The limitation of this study is the retrospective method, performed in only one hospital, the sample is not divided according to period of brain metastasis lesion, history of therapy, no evaluation of adenocarcinoma histological subtype, no special examination from neurologist so minimal symptoms and slight neurological changes can be detected, and the clinical examination performed by different physician.

\section{Conclusion}

Prevalence of lung adenocarcinoma with positive EGFR mutation status of exon 19, exon 21, and negative EGFR mutation status, with brain metastasis in Soetomo General Hospital Surabaya from Janury $1^{\text {st }}$ 2018 until December $31^{\text {st }} 2019$ are $55.9 \%, 48.1 \%$, and $37.0 \%$, respectively.

CT scan and MRI appearance of lung adenocarcinoma with positive EGFR mutation status of exon 19, exon 21, and negative EGFR mutation status are similar with most common location in corticomedullary, parietal lobe, multiple, largest size $1.1-2.5 \mathrm{~cm}$ and $2.6 \mathrm{~cm}$, solid-crotic/cystic component, ring heterogenous contrast enhancement and perifocal edema.

\section{Acknowledgment}

I would like to dedicate my gratitude to all the lecturers of Radiology, Faculty of Medicine Airlangga university, my family, and my friends for their endless support and prayers.

\section{References}

Bahrami E, Taheri M, Benam M. Calcified brain metastatic adenocarcinoma: A case report and review of the literature. Neuroradiol J. 2019 Feb;32(1):57-61. doi: 10.1177/1971400918805184. Epub 2018 Oct 10. PMID: 30303450; PMCID: PMC6327362.

Cruz, C.S., L.T. Tanoue, R.A. Matthay. 2011. Lung cancer : Epidemiology, Etiology, and Prevention. Clin 
Chest Med. 32 (4). DOI: 10.1016/j.ccm.2011.09.001

Lignelli, A., \& Khandji, A. G. 2011. Review of Imaging Techniques in the Diagnosis and Management of Brain Metastases. Neurosurgery Clinics of North America, 22(1), 15-25. DOI:10.1016/j.nec.2010.09.002

Lin, C. Y., C. C. Chang, P. L. Su, C. C. Lin, Y. L. Tseng, W.C. Su, Y. T. Yen. 2019. Brain MRI Imaging Characeristics Predict Treatment Response And Outcome in Patients With De Novo Brain Metastasis of EGFR - Mutated NSCLC. Medicine 98 : 33 (e.16766). DOI: 10.1097/MD.0000000000016765

Mitra, D., Y. H. Chen, R. Li, G. Hermann, K. Atkins, D. Kozono, E. H. Baldini, A. Aizer, U. Chukwueke, R.H. Mak. 2019. EGFR Mutant Locally Advanced Non Small Cell Lung Cancer is at Increased Risk of Brain Metastasis. Clinical and Translational Radiation Oncology. 18 : 32-38. https://doi.org/10.1016/j.ctro.2019.06.008

Pope, W. B. 2018. Brain metastases: neuroimaging. Metastatic Disease of the Nervous System, 89-112. DOI:10.1016/B978-0-12-811161-1.00007-4

Sekine, A., T. Kato, E. Hagiwara, T. Shinohara, T. Komagata, T. Iwasawa et al. 2011. Metastatic Brain Tumors From Non-Small Cell Lung Cancer With EGFR mutation : Distinguishing Influence Of Exon 19 Deletion 10.1016/j.lungcan.2011.12.017

Shin D.Y., I. I. Na, C. H. Kim, S. Park, H. Baek, S. H. Yang. 2014. EGFR Mutation AndBrain Metastasis in Pulmonary Adenocarcinoma. Journal of Thoracic Oncology 9 : 195.199.

Takamori, S., Toyokawa, G., Shimokawa, M., Kinoshita, F., Kozuma, Y., Matsubara, T., ... Maehara, Y. (2018). Radiological Features of Brain Metastases from Non-small Cell Lung Cancer HarboringEGFRMutation. Anticancer Research, 38(6), 3731-3734. doi:10.21873/anticanres.12653

Takano K, Kinoshita M, Takagaki M, et al. Different spatial distributions of brain metastases from lung cancer by histological subtype and mutation status of epidermal growth factor receptor. Neuro Oncol. 2016;18(5):716-724. doi:10.1093/neuonc/nov266

Yoo H., E.Jung, H.S. Gwak, S.H.Shin, S.H.Lee. 2011. Surgical Outcomes of Hemorrhagic Metastatic Brain Tumors. Cancer Research and Treatment: Official Journal of Korean Cancer Association. Vol.43 (2) : $102-107$. DOI : 10.4143/crt.2011.43.2.102

Yousefi, M., T. Bahrami, A. Salmaninejad, R. Nosrati, P. Ghaffari, S. H. Ghaffari. 2017. Lung CancerAssociated Brain Metastasis : Molecular Mechanism And Therapeutic Option. International Society for Cellular Oncology. DOI : 10.1007/s13402-017-0345-5

Zheng Min. 2016. Classification And Pathology of Lung Cancer. Surg Oncol Clin N Am. 25 : 447-468. http://dx.doi.org/10.1016/j.soc.2016.02.003 